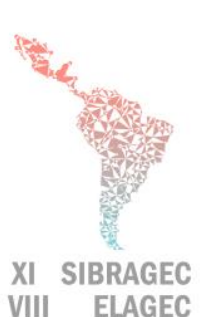

VIII ELAGEC
XI SIMPÓSIO BRASILEIRO DE GESTÃO E ECONOMIA DA CONSTRUÇÃO

VIII ENCUENTRO LATINOAMERICANO DE GESTIÓN Y ECONOMÍA DE LA CONSTRUCCIÓN

Do conhecimento à ação: práticas avançadas de gestão da produção Londrina, Paraná, Brasil. 23 a 25 de Outubro de 2019

\title{
PROPOSIÇÃO DE MODELO DE MATURIDADE PARA SERVIÇOS DE FISCALIZAÇÃO DE OBRAS PÚBLICAS
}

\author{
MATURITY MODEL PROPOSAL FOR PUBLIC WORKS \\ SUPERVISION SERVICES
}

\section{WEBER, Lucas Eduardo (1); CARVALHO, Mayana Chagas (2); FRANCA, Veruschka Vieira (3); SANTOS, Débora de Gois (4)}

(1) Universidade Federal de Sergipe, 79-3194-6937, e-mail: lucas_weber1@live.com (2) Universidade Federal de Sergipe, e-mail: mayanac@gmail.com, (3) Universidade Federal de Sergipe, e-mail: veruschkafanca@gmail.com (4) Universidade Federal de Sergipe, e-mail: deboragois@ yahoo.com.br

\begin{abstract}
Public enterprises play an important role in the social and economic development of society, but their constructions are often characterized by inefficiency in the use of public resources, constant delays in delivery and poor quality. In this context, the construction supervision phase has a great responsibility, as it is one of the phases that contributes to the quality assurance of the public enterprise. The aim of this paper is to propose a maturity model based on the best practices of public entities' inspection teams in order to verify the degree of maturity in relation to the practices established by the TCU. For the exemplification, the proposed model was applied in a public institution, whose key stage analyzed referred to the concreting service and its respective key recommendations. In general, it proved to be effective and easy to apply for the evaluation of maturity in the sector of public works inspections. As a result of the application of the model, it was verified that the institution studied is at the transition level for the mature, with a maturity level of $87 \%$, and the institution places more emphasis on services subject to requests for corrections and technological control.
\end{abstract}

Keywords: Public Works, Supervision, Maturity Model.

\section{INTRODUÇÃOO}

Os contratos públicos, no Brasil, são regidos pela lei 8.666/1993 (BRASIL, 1993) e fiscalizados por órgãos de controle, como o Tribunal de Contas da União (TCU) e Controladoria Geral da União (CGU). Em seu Art. 67 é dito que a execução do contrato público deverá ser acompanhada e fiscalizada por um representante da Administração designado para esta função, sendo permitida a contratação de terceiros para assisti-lo e subsidiá-lo de informações pertinentes a essa atribuição (BRASIL, 1993).

Em 2014, o TCU lançou um guia de "Recomendações Básicas para a Contratação e Fiscalização de Obras de Edificações Públicas" para orientar os fiscais de contratos públicos sobre práticas que devem ser realizadas durante a fiscalização da obra. A fiscalização tem como finalidade verificar o cumprimento das disposições contratuais, técnicas e administrativas em todos os seus aspectos (TCU, 2014). 
Para Gomes (2007), a fiscalização é de vital importância na qualidade de uma obra pública. Não somente no sentido da preservação da autoridade pública ou da punição, mas também no sentido de orientação, para que as especificações e qualidade esperada

sejam cumpridas. Rodrigues (2009) concluiu que além de outros fatores, uma má fiscalização pode causar problemas de qualidade em obras públicas. Zidane e Andersen (2018) apontaram a falta de supervisão como uma das 10 maiores causas de atrasos de obras no mundo.

Diante do exposto, torna-se importante criar um instrumento de avaliação do trabalho de fiscalização de obras públicas, a fim de medir e melhorar seus processos. Uma das possíveis formas de criar esse sistema de avaliação é através de um modelo de maturidade, definido como uma estrutura capaz de quantificar numericamente um desempenho ou uma habilidade com o objetivo de mensurar os pontos fortes e fracos que precisam de melhoria ou identificar oportunidades de aperfeiçoamento (KERZENER, 2006; PRADO, 2008).

O objetivo do artigo consiste em propor um modelo de maturidade com base nas melhores práticas das equipes de fiscalização dos órgãos públicos para verificar o grau de maturidade em relação às práticas estabelecidas pelo TCU em sua obra "Recomendações Básicas para a Contratação e Fiscalização de Obras de Edificações Públicas", publicada em 2014, para ser aplicado em órgãos públicos que fiscalizam obras.

\section{MODELO DE MATURIDADE}

Os modelos de maturidade podem ser usados para apoiar a análise e avaliação de habilidades e níveis de desenvolvimento de produtos e processos (Kluth et al., 2014) e fornecem estrutura para as organizações melhorarem seu desempenho em diferentes áreas (BROOKS et al. 2014).

O Construction Industry Macro Maturity Model (CIM3) foi desenvolvido por Willis e Rankin (2012) e determina pesos e atribui notas na análise da maturidade na construção civil, conforme Equações 1, 2 e 3:

$$
\begin{aligned}
& M=\sum_{i=1}^{12} E C_{i} \\
& E C=\frac{\sum_{i=1}^{n} G R_{i} \cdot P_{i}}{\sum_{i=1}^{n} P_{i}} \\
& G R=\frac{\sum_{x=i}^{n} N_{\chi}}{5 . n}
\end{aligned}
$$

onde,

$$
\begin{array}{ll}
\mathrm{M} & =\text { maturidade; } \\
\mathrm{EC} & =\text { etapa-chave; } \\
\mathrm{P} & =\text { peso; } \\
\mathrm{GR} & =\text { grupo de recomendação-chave; } \\
\mathrm{N} & =\text { nota. }
\end{array}
$$

Assim M representa o nível da classificação de maturidade do objeto de pesquisa em valores percentuais (Figura 1) 
SIBRAGEC - ELAGEC 2019 - de 23 a 25 de Outubro - LONDRINA - PR

Figura 1 - Níveis de maturidade
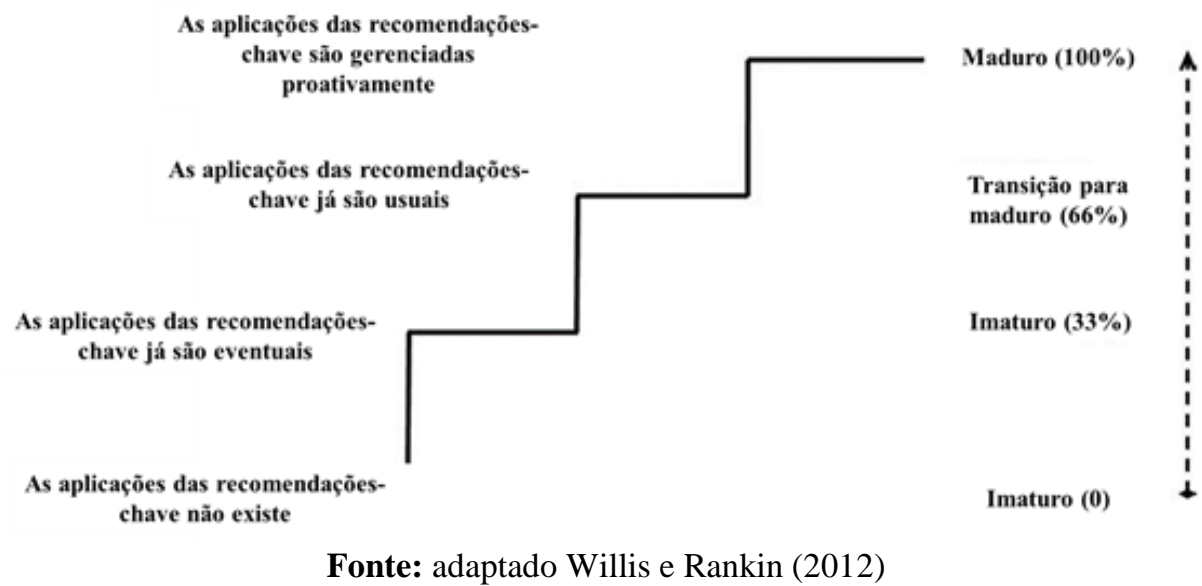

Fonte: adaptado Willis e Rankin (2012)

\section{MÉTODO}

A pesquisa foi descritiva, baseada na elaboração de questionário estruturado, para a construção do modelo de maturidade e aplicação desse com especialistas e em estudo de caso com entrevistas estruturadas (Figura 2).

Figura 2 - Fluxograma do método de pesquisa

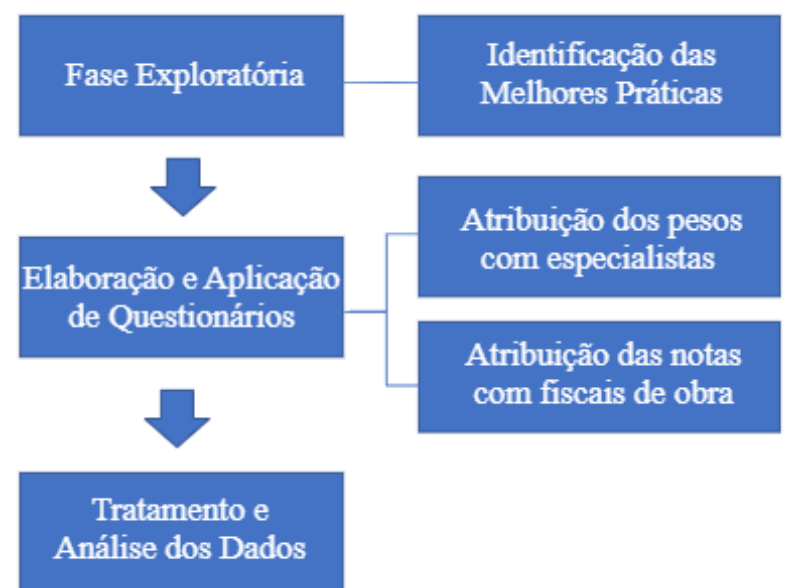

Fonte: Autores (2019)

\subsection{Concepção do modelo de maturidade}

De acordo com o guia do TCU (2014), a fase de fiscalização de obras é composta por 12 etapas, que no modelo proposto equivale às 12 etapas-chave (EC). Para cada uma dessas etapas, o guia estabelece um conjunto de recomendações, consideradas como recomendações-chaves (RC), que devem ser seguidas pela equipe de fiscalização do órgão (Figura 3). 
SIBRAGEC - ELAGEC 2019 - de 23 a 25 de Outubro - LONDRINA - PR

Figura 3 - Esquema geral do modelo de maturidade proposto

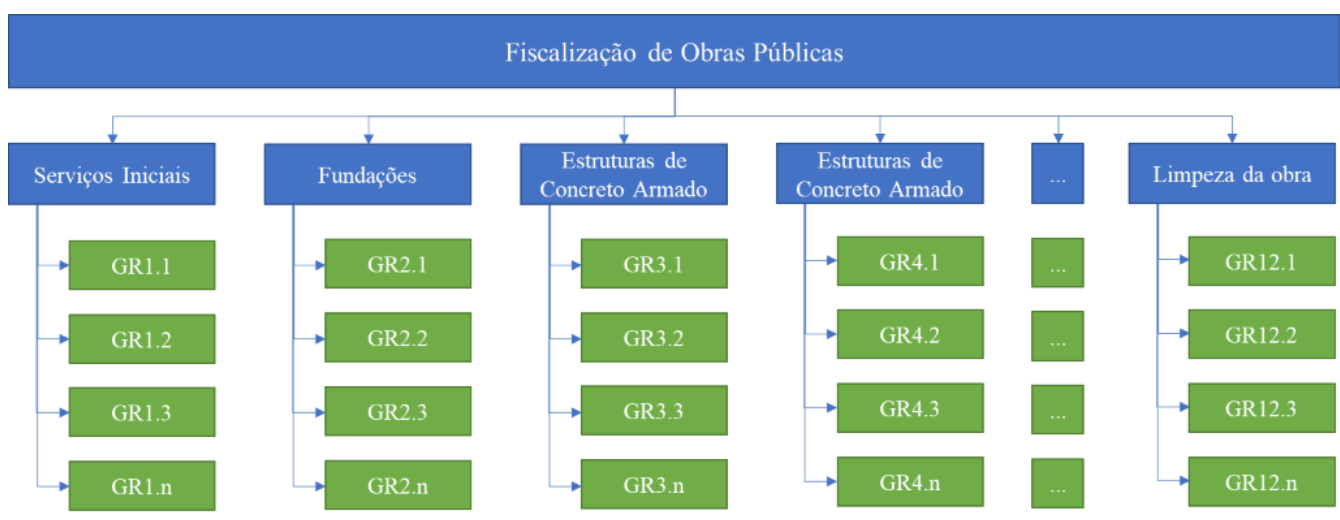

Fonte: Autores (2019)

Para a criação da matriz de prioridades, em cada EC devem ser elaborados e realizados os testes pilotos de dois questionários em escala de Likert (1 a 5). O primeiro para ser aplicado aos especialistas na área, onde obtêm-se os pesos para o grupo de cada recomendação (GR) e o segundo, à equipe de fiscalização de obras para que sejam atribuídas notas para cada RC (Figura 4).

Figura 4 - Esquema para aplicação de pesos e notas

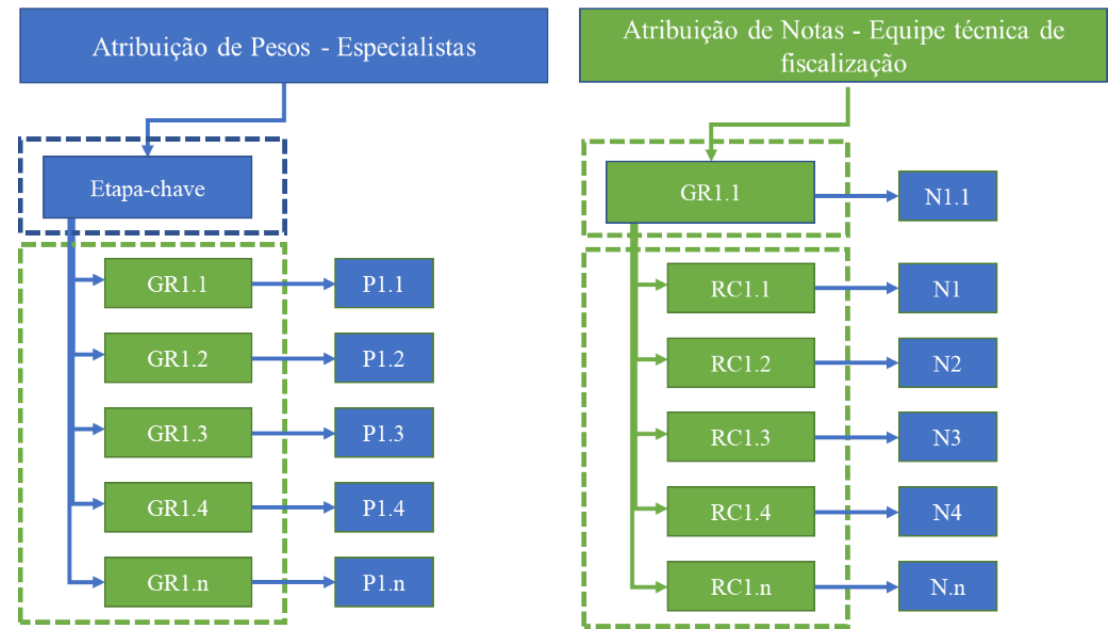

Fonte: Autores (2019)

\subsection{Aplicação do modelo}

Para a exemplificação, o modelo proposto foi aplicado em uma instituição pública no setor de fiscalização de obras. A EC analisada referiu-se ao serviço de estrutura de concreto armado (CA), bem como às suas respectivas RC (Figura 5). 
SIBRAGEC - ELAGEC 2019 - de 23 a 25 de Outubro - LONDRINA - PR

Figura 5 - Exemplificação do modelo proposto

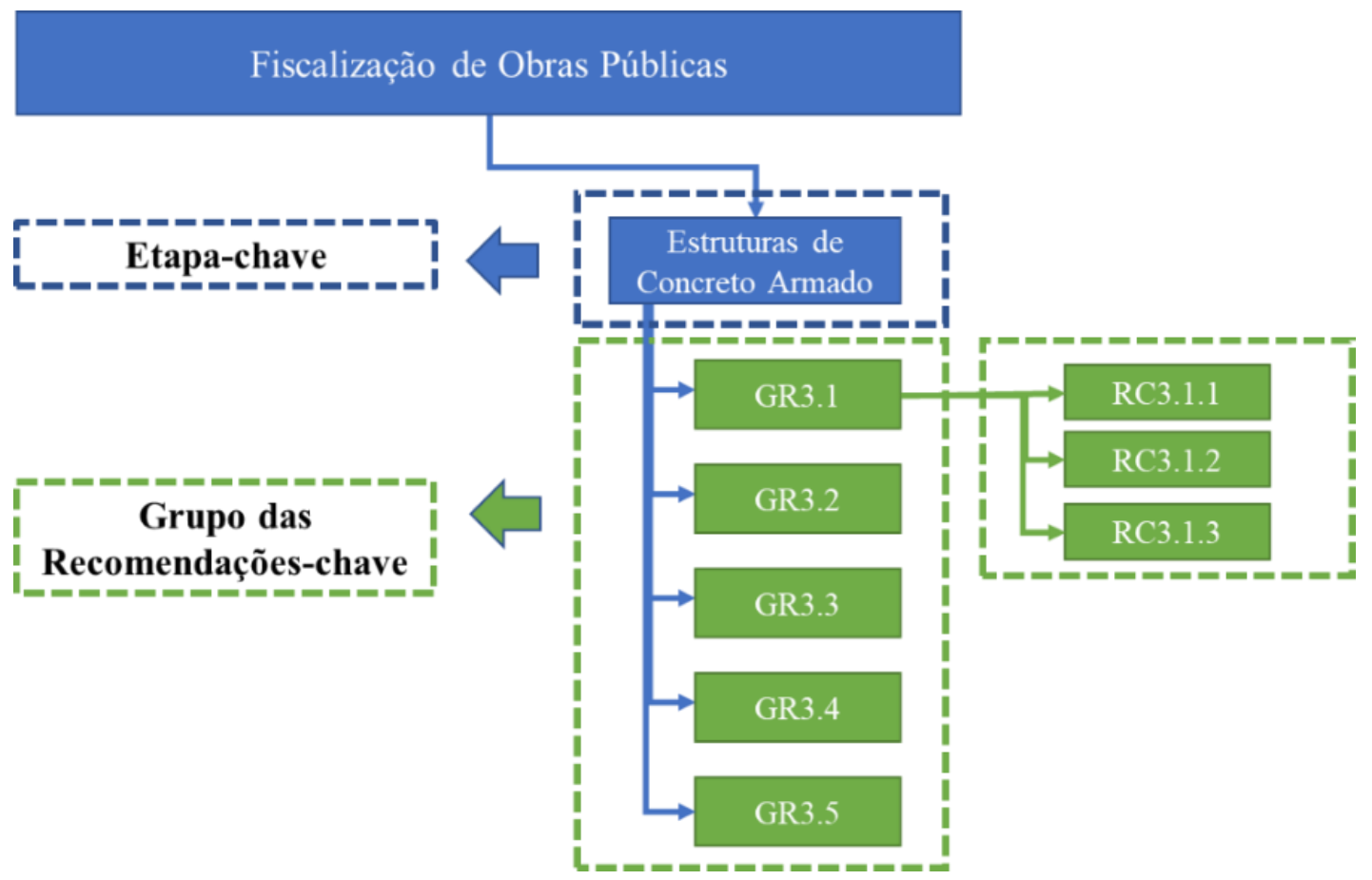

Fonte: Autores (2019)

Originalmente, para esta etapa-chave, o TCU (2014) determinou um conjunto de 6 recomendações, que foram agrupadas em cinco GR (Quadro 1).

\section{Quadro 1 - Agrupamento das recomendações do TCU (2014)}

\begin{tabular}{|c|l|}
\hline Grupos & \multicolumn{1}{|c|}{ Recomendações } \\
\hline $\begin{array}{c}\text { Conferência prévia } \\
\text { (GR3.1) }\end{array}$ & $\begin{array}{l}\text { Realizar a conferência dos serviços e materiais utilizados antes da } \\
\text { execução da concretagem; }\end{array}$ \\
\hline $\begin{array}{c}\text { Acompanhamento } \\
\text { (GR3.2) }\end{array}$ & $\begin{array}{l}\text { Realizar o acompanhamento do serviço de concretagem, a fim de verificar } \\
\text { se as normas/boas práticas estão sendo cumpridas; }\end{array}$ \\
\hline Controle (GR3.3) & $\begin{array}{l}\text { Solicitar que seja feito o controle tecnológico do material empregado no } \\
\text { serviço; }\end{array}$ \\
\hline $\begin{array}{c}\text { Conferência pós } \\
\text { execução (GR3.4) }\end{array}$ & Realizar a conferência do serviço após sua execução; \\
\hline Correções (GR3.5) & $\begin{array}{l}\text { Solicitar as correções caso seja detectado algo em desacordo com o } \\
\text { projeto ou mal executado. }\end{array}$ \\
\hline
\end{tabular}

Fonte: adaptado TCU (2014)

Em seguida, foram elaborados e aplicados dois conjuntos de questionários: o primeiro, para a atribuição de pesos pelos especialistas (Quadro 1); e o segundo, para estabelecer o grau de importância das recomendações-chave sob a percepção dos servidores responsáveis pela fiscalização de obras do órgão escolhido (Quadro 2). 
SIBRAGEC - ELAGEC 2019 - de 23 a 25 de Outubro - LONDRINA - PR

\section{Quadro 2 - Recomendações-chave para estruturas de concreto armado}

\begin{tabular}{|c|c|}
\hline Grupos & Recomendações \\
\hline \multirow[t]{3}{*}{ GR3.1 } & $\begin{array}{l}\text { 3.1.1 É realizada a verificação das dimensões, alinhamento, posicionamento, } \\
\text { vedação, travamento e limpeza das formas antes da realização da concretagem da } \\
\text { peça. }\end{array}$ \\
\hline & $\begin{array}{l}\text { 3.1.2 Antes de concretar uma estrutura, o posicionamento e as bitolas das ferragens } \\
\text { são conferidos de acordo com o projeto. }\end{array}$ \\
\hline & $\begin{array}{l}\text { 3.1.3 Os eletrodutos, dutos e demais instalações são conferidos quanto à sua } \\
\text { dimensão e localização antes de se iniciar a concretagem. }\end{array}$ \\
\hline \multirow{4}{*}{ GR3.2 } & $\begin{array}{l}\text { 3.2.1 As recomendações técnicas sobre preparo e transporte são observadas durante } \\
\text { o acompanhamento do serviço de concretagem. }\end{array}$ \\
\hline & 3.2.2 É verificado se a contratada faz a vibração do concreto durante a sua execução. \\
\hline & $\begin{array}{l}\text { 3.2.3 É solicitado que a contratada mantenha uma rotina de cura de concreto após a } \\
\text { concretagem }\end{array}$ \\
\hline & 3.2.4 É verificado se a empresa cumpre os prazos de desforma. \\
\hline GR3.3 & $\begin{array}{l}\text { 3.3.1 São cobrados os ensaios de controle tecnológico dos materiais utilizados na } \\
\text { concretagem. }\end{array}$ \\
\hline \multirow{2}{*}{ GR3.4 } & $\begin{array}{l}\text { 3.4.1 Após a concretagem, é verificado os prumos nos cantos externos, pilares, } \\
\text { poços de elevadores ou algum outro ponto principal da obra. }\end{array}$ \\
\hline & 3.4.2 É conferido se a junta de dilatação obedece ao detalhe do projeto. \\
\hline GR3.5 & $\begin{array}{l}\text { 3.5.1 Caso ocorra alguma falha durante a concretagem, é solicitado que a empresa } \\
\text { faça as devidas correções para que a estrutura fique condizente com o projeto. }\end{array}$ \\
\hline
\end{tabular}

Fonte: adaptado TCU 2014.

Por fim, com os dados obtidos, foram aplicadas as equações detalhadas na seção 2.1 deste artigo e foi determinado o nível de maturidade para a etapa-chave de estruturas de concreto para o setor de fiscalização da instituição pública analisada.

\section{RESULTADOS}

Na primeira etapa de aplicação dos questionários, foram consultados 5 especialistas: 2 engenheiros fiscais de obras com 10 anos de experiência cada, sendo um mestre e outro doutor; 3 engenheiros civis, professores de ensino superior sendo 1 mestre e perito com 28 anos de experiência, 1 doutor com 32 anos de experiência e outro doutor com 10 anos de experiência como projetista de estruturas.

Com base nos dados coletados e organizados, foram calculadas as médias aritméticas por grupo e foram encontrados os resultados referentes ao peso (Quadro 3).

Quadro 3 - Pesos aplicados às recomendações-chave

\begin{tabular}{|l|c|c|c|c|c|c|}
\hline \multicolumn{1}{|c|}{ Grupos } & E1 & E2 & E3 & E4 & E5 & Pesos \\
\hline Conferência Prévia & 5,0 & 5,0 & 5,0 & 4,0 & 5,0 & 4,8 \\
\hline Acompanhamento & 3,0 & 4,0 & 5,0 & 5,0 & 5,0 & 4,4 \\
\hline Controle Tecnológico & 4,0 & 5,0 & 5,0 & 5,0 & 4,0 & 4,6 \\
\hline Conferência Pós Execução & 3,0 & 4,0 & 4,0 & 4,0 & 4,0 & 3,8 \\
\hline Correções & 4,0 & 5,0 & 5,0 & 4,0 & 4,0 & 4,4 \\
\hline
\end{tabular}

Fonte: Autores (2019) 
SIBRAGEC - ELAGEC 2019 - de 23 a 25 de Outubro - LONDRINA - PR

Em relação à segunda etapa, foram consultados 5 servidores da instituição pública analisada, sendo 4 engenheiros civis com experiência entre 4 e 10 anos e 1 técnico em edificações com experiência de 4 anos. Com base nos dados coletados e organizados, foram calculadas as médias aritméticas de cada recomendação-chave e a média aritmética para cada grupo. Já para o cálculo da média relativa, foi utilizada a equação 3 e os resultados agrupados no Quadro 4.

Quadro 4 - Nota da recomendação-chave

\begin{tabular}{|c|c|c|c|c|}
\hline \multicolumn{2}{|c|}{ Grupos } & $\begin{array}{c}\text { Média Aritmética } \\
\text { das Notas }\end{array}$ & $\begin{array}{c}\text { Média Aritmética } \\
\text { do Grupo }\end{array}$ & Média Relativa \\
\hline \multirow{3}{*}{ GR3.1 } & 3.1 .1 & 4,0 & \multirow{3}{*}{4,4} & \multirow{3}{*}{0,88} \\
\hline & 3.1 .2 & 5,0 & & \\
\hline & 3.1 .3 & 4,2 & & \\
\hline \multirow{4}{*}{ GR3.2 } & 3.2 .1 & 3,2 & \multirow{4}{*}{4,1} & \multirow{4}{*}{0,82} \\
\hline & 3.2 .2 & 4,8 & & \\
\hline & 3.2 .3 & 4,2 & & \\
\hline & 3.2 .4 & 4,2 & & \\
\hline GR3.3 & 3.3 .1 & 4,8 & 4,8 & 0,96 \\
\hline \multirow{2}{*}{ GR3.4 } & 3.4 .1 & 3,0 & \multirow{2}{*}{3,4} & \multirow{2}{*}{0,68} \\
\hline & 3.4 .2 & 3,8 & & \\
\hline GR3.5 & 3.5 .1 & 5.0 & 5,0 & 1,00 \\
\hline
\end{tabular}

Fonte: Autores (2019)

Com esses resultados foi traçado um gráfico (Figura 6) que demonstra visualmente as médias das notas atribuídas pelos servidores.

Figura 6 - Gráfico com os resultados notas das recomendações-chave

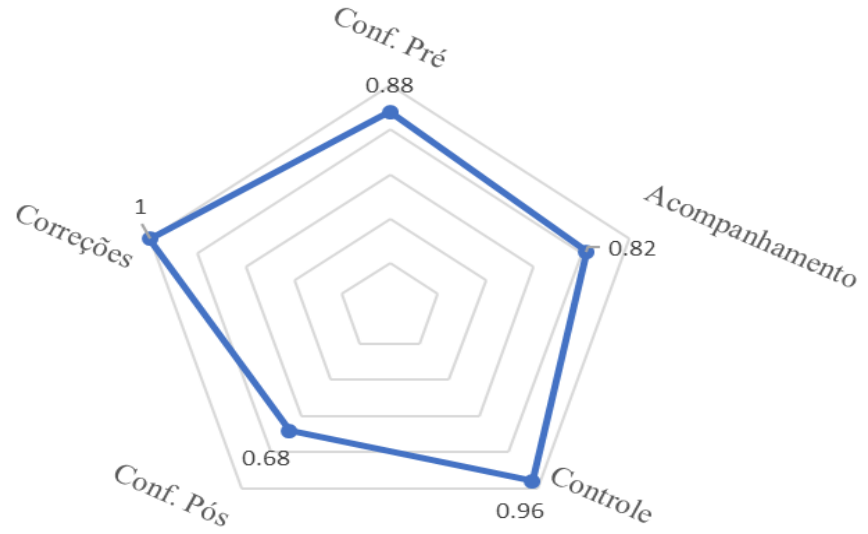

Fonte: Autores (2019)

Ao final, com os pesos e notas definidos foram calculados, através da Equação 2, a média ponderada que representa a etapa-chave, conforme demostrado na Equação 4:

$$
E C_{E s t . C A}=\frac{0.88 \times 4.8+0.82 \times 4.4+0.96 \times 4.6+0.68 \times 3.8+1.0 \times 4.4}{(4.8+4.4+4.6+3.8+4.4)}=0.87
$$


Como a matriz de maturidade foi aplicada somente para um serviço, ao aplicar a Equação 1 , obtém-se o resultado de 0.87 . Isso significa que o resultado referente ao nível de maturidade é de $87 \%$, ou seja, o setor de fiscalização da instituição pública exemplificada para o serviço de fiscalização de Estruturas de CA encontra-se no nível de transição para maduro, conforme Figura 1.

A Figura 7 demonstra os valores percentuais máximos que poderiam ser obtidos após a aplicação dos pesos de cada recomendação e os valores obtidos na instituição onde foi aplicado o modelo.

Figura 7 - Gráfico das notas ponderadas da instituição

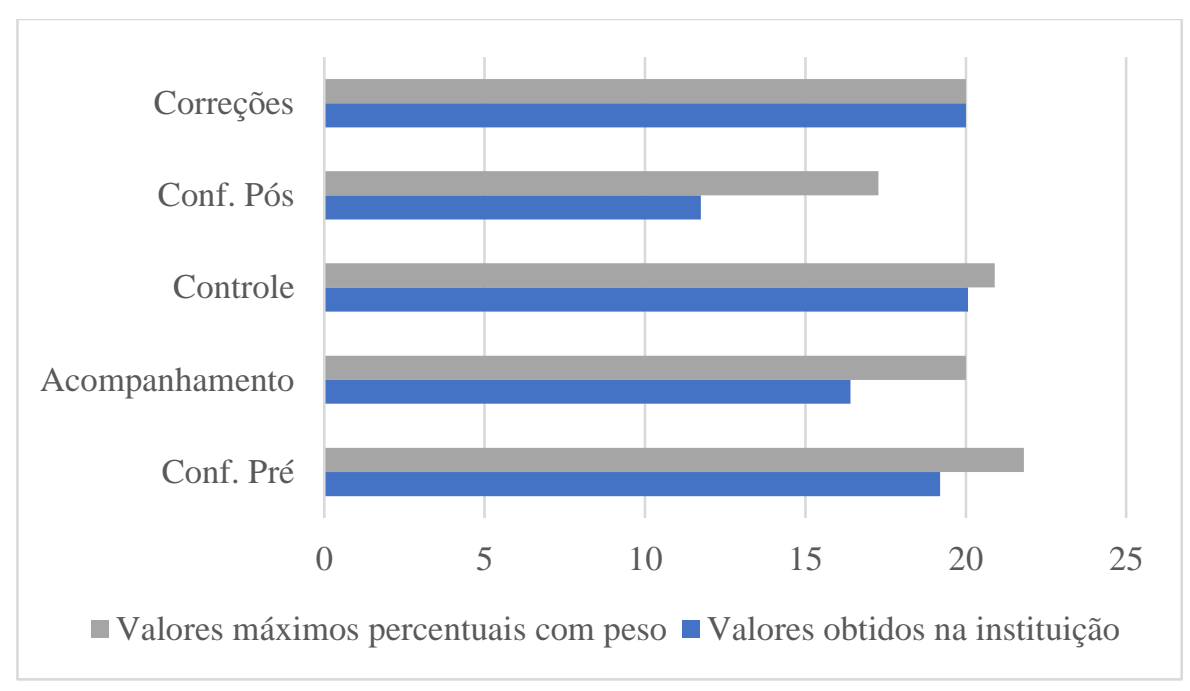

Fonte: Autores (2019)

Através das Figuras 6 e 7, é possível verificar que há a possibilidade de evolução do órgão em relação aos grupos de RC ligadas principalmente à conferência pós-execução. As recomendações agrupadas em serviços de conferência prévia, acompanhamento e controle tecnológico obtiveram bom resultado, mas é possível amadurecer ainda mais o conhecimento do órgão estudado nessas áreas. Em contrapartida, as recomendações ligadas às correções estão totalmente maduras.

Contudo, o resultado obtido demonstra que a instituição analisada é menos madura exatamente na área que tem o menor peso de importância para a garantia da qualidade do serviço de Estrutura de Concreto Armado.

\section{CONSIDERAÇÕES FINAIS}

O modelo apresentado mostrou-se eficaz e de fácil aplicação para a avaliação da maturidade no setor de fiscalizações de obras públicas, pois o mesmo pode ser replicado em qualquer instituição que realiza esse serviço, a fim de melhorarem o seu desempenho.

Após a aplicação do modelo, verificou-se que a instituição estudada se encontra no nível de transição para o maduro em relação ao serviço de estruturas de concreto armado, com um nível de maturidade de $87 \%$. Observou-se que a instituição dá mais ênfase nos serviços referentes à solicitação de correções e controle tecnológico. 
SIBRAGEC - ELAGEC 2019 - de 23 a 25 de Outubro - LONDRINA - PR

\section{REFERÊNCIAS}

BRASIL. Lei n.. 8666, de 21 de junho de 1993. Institui normas para licitações e contratos da Administração Pública, e dá outras providências. Disponível em:

<http://www.planalto.gov.br/ccivil_03/leis/18666cons.htm> Acesso em: 12 dez. 2018.

BROOKES, N.; BUTLER, M.; DEY, P.; CLARK, R. The use of maturity models in improving project management performance: An empirical investigation. International Journal of Managing Projects in Business, v. 7, n. 2, p. 231-246, abr. 2014.

GOMES, R. C. G. G. A postura das empresas construtoras de obras públicas da grande Florianópolis em relação ao PBQP-H. 2007. Dissertação (Mestrado em Engenharia Civil) Programa de Pós-Graduação em Engenharia Civil, Universidade Federal de Santa Catarina, Florianópolis, 2007.

KERZENER, H. Gestão de projetos: as melhores práticas (2a ed.). Porto Alegre: Bookman, 2006.

KLUTH, A.; JAGER, J.; SCHATZ, A.; BAUERNHANSL, T. Evaluation of Complexity

Management Systems - Systematical and Maturity-Based Aproach. Procedia CIRP, v. 17, n. 1, p. 224-229, 2014.

PRADO, D. S. Maturidade no gerenciamento de projetos. Nova Lima: INDG Tecnologia e Serviços Ltda., 2008.

RODRIGUES, A. A. Qualidade na contratação e gerenciamento de obras públicas. 2009. Trabalho de conclusão de curso (Especialização em auditoria de obras públicas) - Pontifícia Universidade Católica do Rio de Janeiro, Rio de Janeiro, 2009.

TCU. Tribunal de Contas da União. Obras Públicas: Recomendações básicas para a contratação e fiscalização de obras de edificações públicas. 4. ed. Brasília: TCU, Secretaria Geral de Controle Externo, Secretaria de Fiscalização de Infraestrutura Urbana, 2014.

WILLIS, J. C.; RANKIN, J. H. The Construction Industry Macro Maturity Model (CIM3): theoretical underpinnings. International Journal of Productivity and Performance

Management, v. 61, n. 4, p. 382 - 402, 2012.

ZIDANE, Y. J. T; ANDERSEN, B. The top ten universal delay factors in construction projects.

International Journal of Managing Projects in Business, v. 1, n. 3, p. 650-672, 2018.

\section{AGRADECIMENTOS}

Aos entrevistados pela atenção conferida e à CAPES. 